\title{
REGENERATION IN THE HUMAN SPINAL CORD: A REVIEW OF THE RESPONSE TO INJURY OF THE VARIOUS CONSTITUENTS OF THE HUMAN SPINAL CORD*
}

\author{
By J. Trevor Hughes, M.D., F.R.C.P., F.R.C.Path. \\ Dept of Neurophathology, Radcliffe Infirmary, Oxford OX2 6HE, England
}

IT is now 25 years since I began the study of the pathology of the human spinal cord in injury and disease. During that time I have examined pathologically the necropsies of some hundreds of human cases of spinal cord injury and have also participated in the histological examination of the tissues arising from experimental injury to the spinal cord. Like many others before me and many more to come I have been fascinated by the problem of regeneration in the human spinal cord and this is a subject about which I am frequently asked. I am pleased to participate in this Meeting of the International Medical Society of Paraplegia and to discuss some of the problems concerned with regeneration in the spinal cord. My remarks are mainly based on my own observations, which have been published in detail elsewhere (Hughes, I 974 and 1978), although I shall refer occasionally to the works of others on human spinal cord regeneration and to some experimental work.

\section{Definition of the Problem}

The question I am asking is 'How much effective regeneration of the anatomical structures and the physiological mechanisms occurs in the adult human spinal cord after injury?' I so frequently attend conferences in which the speakers are thought to be referring to this subject but are presenting clinical, physiological or anatomical findings on another topic.

The species involved is all important. Regeneration of tissues including the nervous system becomes more complete as one descends the scale of complexity in the animal kingdom. Homo sapiens, judged by his central nervous system, is the most complex and highly developed species of all. The ability of the central nervous system in man to regenerate after trauma is probably less than in sub-human primates. If we study regeneration of the nervous system in other vertebrates such as dog, cat, sheep and rodents the experimental results cannot be taken as valid for man. The central nervous system is less complex in several ways. An example of this is the much simpler forms of glia present in the mouse, rat and other rodents. Another very important feature is the ability of the spinal cord in some 'lower' animals to take on an independent role after it has become detached from the brain.

A feature of great importance is the age of the experimental animal. In the consideration of spinal cord regeneration in human cases, age is only

\footnotetext{
*This was an Invited Lecture, given during the Annual Meeting of the International Medical Society of Paraplegia, Stoke Mandeville Hospital, Aylesbury, Bucks, England, May 1983.
} 
of slight importance because in most cases the nervous system has achieved maturity. In many experimental animals, the subsequent growth within the central nervous system can be confused with regeneration after trauma.

\section{Does Regeneration Occur in the Human Spinal Cord?}

The short answer to this question is 'yes' but we must be extremely careful in defining with precision the anatomical tissues we are considering. Regeneration of the spinal meninges and of the spinal vessels and supporting connective tissues occurs. Regeneration of glia occurs but is incomplete. Regeneration of certain spinal neurones and their axons can be extensive but there are important features which prevent this regeneration being effective.

\section{Spinal Meninges}

The dura mater forms a loose sack containing the spinal cord. It is very strong and has great regenerative potential. Following spinal cord injury, at necropsy several months or years later the spinal dura is largely intact. It may have been contused or lacerated but, if survival was sufficiently long, this damage is repaired and there is little more to be seen than some unobtrusive connective tissue thickening. This is the appearance following contusions even with gross trauma and also that seen after minor tears and puncture wounds. If the dura mater is completely torn across and there is permanent displacement of the two cut ends of the spinal dural sack then healing will be incomplete. The final state of the spinal cord wound ends in a connective tissue scar in which the meninges are blended with the connective tissue scar replacing the spinal cord. This state is even more likely to follow if there has been infection at the site of trauma or the persistence of foreign bodies or displaced bone.

The arachnoid mater and the pia mater although much thinner than the dura are probably equally strong. They resist tearing in all but gross trauma and are capable of considerable repair. In contusion of the spinal cord of severe degree the arachnoid and pia are reconstituted to a normal appearance. Tears and lacerations are repaired completely and even short distances of up to $0.5 \mathrm{~cm}$ seemed to be bridged by new growth of both pia and arachnoid.

\section{Spinal Vessels and Connective Tissues}

As one might expect from their connective tissue origin, all the spinal vessels, whether arteries, veins or capillaries are capable of repair after injury. The spinal vasculature is of great importance in understanding the damage caused to the spinal cord during injury, but it itself survives all but gross spinal trauma. It is quite usual to find that the spinal cord has been completely destroyed and converted into a connective tissue and glial scar in which only a ghost outline of its former structure remains. Despite this complete parenchymal destruction one may find a normal anterior spinal artery and vein but serving not a spinal cord but a connective tissue scar replacing the spinal cord. Examination of cases at varying stages after injury shows important histological changes in the spinal vessels particularly the 
medium sized arteries such as the anterior and posterior spinal arteries. It is clear however that most if not all of the spinal vessels remain patent and are presumably remodelled in size to serve the surviving tissues. From this it can be deduced that whereas at the time of injury the effectiveness of the blood supply might be an important factor in causing the spinal cord damage, subsequently the spinal vessels are not the reason for the failure of effective regeneration.

The role of connective tissue formed from fibroblasts requires more careful consideration. When there is moderate damage to the human spinal cord there may be a complete spinal cord transection and subsequently the spinal cord shows loss of all neurones and axons and their replacement by glia made up of astrocytes and their fibrous processes. There is very.little connective tissue to be seen other than a slight thickening of the normal connective tissue architecture based on the intramedullary spinal vessels. If, however, the spinal cord damage is more severe and accompanied by intramedullary blood clot, or foreign material, or most importantly infection, then the destructive process destroys the glia as well as the neurones, axons and myelin. The result is a connective tissue scar instead of a glial scar. Tracing the lesion up or down the cord one can see the transition between a connective tissue scar in the central part of the spinal cord trauma to a glial scar above and below and finally reaching intact cord apart from the Wallerian degeneration of the long tracks proceeding upwards and downwards. Whether the connective tissue scar helps or hinders regeneration is controversial. My own feeling is that it is the absence of the glia and not the presence of the connective tissue which is important. Regenerating axons seem to be able to grow readily in connective tissue. The problem seems to be that in this location they acquire a myelin sheath of peripheral myelin type derived from the Schwann cell rather than a myelin sheath of the central type derived from oligodendroglia.

\section{The Role of Neuroglia in Regeneration}

The various types of neuroglia are astrocyte, the oligodendrocyte, the microglia and the ependymal cell. In the adult spinal cord the ependyma is small in amount and now has no obvious function. It will however regenerate rapidly and will for example line a syringomyelic cavity. The ependymal growth in this location is similar to its behaviour in an open central canal or in the lining of the ventricles of the brain.

The microglial cell, resting in the adult spinal cord, becomes enlarged after trauma and serves a phagocytic function. These 'resting' microglia are added to by numerous macrophages entering the spinal cord from the blood vessels. The function of the microglia and of the macrophages from the blood appears to be that of the disposal of breakdown products. There is no known function of the microglia in any regenerative phenomenon.

The astrocyte is a glial cell of great complexity and about whose function we still know very little. Astrocytes are present in great matter and in white matter and there are two types, fibrous and protoplasmic. The fibrous type occurs everywhere but the protoplasmic type occurs only in grey matter. The astrocyte has an important supportive role in the central nervous tissues. Its other role is probably that of nutrition of neurones and their processes. Whether the astrocyte will replace itself by multiplica- 
tion of survivors after loss from injury is still debatable. It is said that mitoses are not seen in astrocytes in the adult central nervous system. I do not think that this is known with certainty for the human spinal cord. It is quite difficult to identify the nature of a cell undergoing mitosis. What is known is that astrocytes enlarge considerably after trauma. Their fibrous processes develop, elongate and branch, and a mossy network of fibres results. This network replaces the absent neurones and axons. We must leave the subject of the astrocyte with the recommendation that further research into the function of this cell is of the utmost importance in the understanding of our problems of spinal cord regeneration.

The structure and function of the oligodendrocyte is now largely understood. From the parent cell body with a small round nucleus, cytoplasmic processes, which may number up to 40 wind themselves around the axons forming the myelin sheath of the central nervous axon. In the developing nervous system the myelin is in continuity with the cell body and this anatomical relationship persists into adult life. It is now clear that in certain limited circumstances, if the myelin sheath is lost from central nervous axons, limited repair can occur. This probably occurs by the regrowth of a new process from a persisting oligondendroglial cell. As with the astrocyte, it is difficult to decide whether there is replication of oligodendrocytes after injury. Mitotic figures are said not to occur in oligodendrocytes but this question is difficult of solution for the reasons given in discussing the astrocyte.

\section{Regeneration of Spinal Neurones}

We now come to the very important question of whether spinal neurones regenerate after damage. At once we must distinguish between the various types of neurones encountered in the spinal cord. They may be grouped into four although obviously many further sub-divisions could be made. These four groups are: the spinal motor neurones, the neurones of the peripheral sensory ganglia, the long intra-spinal neurones, and the short inter-nuncial neurones. These will be considered in turn but first we must discuss whether we are considering replacement of neurones or repair of damaged neurones.

\section{Replacement of Lost Spinal Neurones}

All the evidence so far is that in man a central nervous system neuron is not replaced if it is lost by trauma or disease. This conclusion is probably true for all the large neurones that have been studied. For small internuncial neurones it is doubtful whether the relevant observations have yet been made but probably the same rule applies to small neurones as to large neurones. We shall state this again-Loss of neurones in the adult human spinal cord is irrevocable. This loss is not always as serious a problem as may be thought. It would of course affect the segment of spinal cord involved in trauma and at the cervical enlargement or the lumbosacral expansion would result in serious neurological deficit. However, if the long tracks could be reconstituted the loss of function from, for example, two segments of the thoracic spinal cord could be sustained by the otherwise paraplegic person without great disability. Having dealt with the problem 
of replacement of lost neurones; the current opinion is that this is impossible, we can now discuss the repair of the severed process of a neuron.

\section{Spinal Motor Neurones}

The spinal motor neuron has its cell body in the anterior horn of the spinal cord. The main axon proceeds through the spinal cord for a short distance to the anterior spinal nerve root exit zone and here it changes from a central nervous axon to an axon of the peripheral nervous system. At this important transition zone it gives up its central myelin sheath derived from oligodendroglia and takes on a peripheral myelin sheath with Schwann cells. When the axon from this neuron is severed, regeneration is not only possible but occurs invariably. In a different location this is seen in peripheral nerve injuries forming the familiar neuroma in cases proceeding badly and the reconstituted peripheral nerve in fortunate injuries or cases treated well. The distance of the injury to the peripheral nerve from the parent cell body in the anterial horn is important. Regeneration with restoration of function is more complete in a limb than in a plexus, and in a plexus than in the spinal root central to the plexus. Within the spinal cord regeneration also occurs but the fibres are more likely to be directed wrongly. If they do not exit correctly they may turn into the spinal cord and form a small neuroma. These fibres are clothed with peripheral myelin derived from Schwann cells even though they are within the spinal cord.

\section{Neurones of the Peripheral Sensory Ganglia}

These are bipolar neurones with a peripheral process entering the peripheral nerve and a central process entering the spinal cord via the posterior nerve root. Within the spinal cord this central process divides and its long process ascends in the posterior columns to the brain stem. Damage to this neuron is capable of repair but we must be precise in considering which part of the neuron is damaged and regrows. The peripheral process of the neuron regrows in a similar manner to that described for the motor neuron. This also happens to the central process as far as its entry into the spinal cord. If these axons are severed, as for example in a neurosurgical rhizotomy, regrowth occurs sometimes into the subarachnoid space but also sometimes into the spinal cord. This regrowth phenomenon is seen in spinal cord diseases as well as in spinal trauma. In certain cases of trauma and in certain spinal diseases the whole of many segments of spinal cord are full of the fibres regrowing from the posterior roots (Hughes and Brownell, I963). These fibres are clothed with peripheral myelin derived from Schwann cells. They do not grow in any orderly way but in a matted network of fibres resembling a skein of wool. In the scar of the spinal trauma the fibres turn back above and below thus not entering the glial region above and below the connective tissue scar. When we look at the posterior columns some way above the spinal cord transection with axon or myelin stains it is clear that no regeneration of the central processes of these neurones has occurred. In the posterior columns of these cases there is a clearly marked zone of absent axons and myelin forming a white unstained area of tract degeneration. Earlier in time with Marchi preparations this area will be picked out because of the degenerating myelin. In 
a Nauta stain or in other similar axon stains applicable to cases examined soon after injury, the axonic degeneration in the posterior columns above a transection can be seen.

\section{Long intra-spinal Neurones}

There are several long intra-spinal neurones of which the most obvious example are the neurones of the posterior spino-cerebellar tracts. The cell bodies are in Clarkes column in the grey matter of the lateral horn of the thoracic spinal cord segments. The main axons proceed upwards in the posterior spino-cerebellar track and end in the medulla. When lost by trauma or disease these neurones are not replaced. When their axons are severed the typical Wallerian degeneration is seen caudal to the transection. Axons and myelin are lost, and the tract is outlined merely by gliosis. In the human spinal cord there is no regeneration of this neuron. The abortive regeneration with the appearance of peripheral myelin formed by Schwann cells is not seen in this neuron. Presumably similar considerations apply to other long intra-spinal neurones such as those of the rubro-spinal and tecto-spinal tracts.

\section{Inter-nuncial Neurones}

Research using methods of neurophysiology continually expands the function of small neurones whose activity is related to the larger neurones which are more obvious and more easily studied. An obvious example of these small neurones are the Renshaw cells which are small neurones in the anterior horns. We know nothing about whether these neurones are replaced if lost by trauma or disease. Nor do we know whether a part of the processes of these neurones can be regenerated. These neurones are concerned with forming synapses on other neurones. It is quite likely that synapses can be reformed and this plasticity in forming new synapses in the nervous system is probably of considerable importance.

I have now completed this review of the regeneration of the different constituents of the central nervous system within the spinal cord and propose to summarise my conclusions and speculations as follows.

\section{Spinal Meninges}

The dura, arachnoid, and pia mater are regenerated easily after minor trauma and substantial repair is possible after major trauma.

\section{Spinal Vessels and Connective Tissues}

Damage to spinal vessels is important in the acute stage of trauma. Subsequently these vessels are repaired or regenerate forming an excellent blood supply to the spinal cord scar. Connective tissues formed from the fibroblasts regenerate well but may be a hinderance to complete recovery.

\section{Neuroglia}

Ependyma and microglia regenerate well. Astrocytes and oligodendrocytes may not be replaced if lost but have potential for hypertrophy and repair. 


\section{Spinal Neurones}

Spinal motor neurones and the neurones of the peripheral sensory ganglia regenerate their peripheral processes but not their central processes after their entry into the spinal cord. Abortive regeneration of central or peripheral processes into the spinal cord is common and has caused great confusion in the understanding of the subject of regeneration. Long intraspinal neurones (e.g. those of the spino-cerebellar tracks) are not regenerated. We have no knowledge of replacement or regeneration of the smaller internuncial neurones of the spinal cord.

\section{REFERENCES}

Hughes, J. T. (1974). Pathology of spinal cord trauma. Chapter $2 \mathrm{I}$ in 'Brock's Injuries of the Brain and Spinal Cord' Fifth edition, edited by E. H. Feiring. Springer Publishing Co. Inc., New York.

Hughes, J. T. (1978). Pathology of the Spinal Cord. 2nd edition. Lloyd-Luke, London. Hughes, J. T. \& BRownell, B. (1963). Aberrant nerve fibres within the spinal cord. F. Neurol. Neurosurg. and Psychiat., 26, 528-534. 